\title{
Functional Connectivity of the Medial Temporal Lobe Relates to Learning and Awareness
}

\author{
Anthony Randal McIntosh, ${ }^{1}$ M. Natasha Rajah, ${ }^{1}$ and Nancy J. Lobaugh ${ }^{2}$ \\ ${ }^{1}$ Rotman Research Institute of Baycrest Centre, University of Toronto, Toronto, Ontario M6A 2E1, Canada, and 2Sunnybrook and Women's College Health \\ Sciences Centre, University of Toronto, Toronto, Ontario, M4N 3M5, Canada
}

Learning with awareness is believed to require the involvement of the medial temporal lobe (MTL). In this study, the hypothesis tested was that this involvement is best appreciated by the pattern of MTL functional connectivity with other brain areas. In a sensory learning paradigm, human subjects were classified as AWARE or UNAWARE, on the basis of whether they noted that one of two tones predicted a visual event. Only AWARE subjects acquired and reversed a differential response to the tones. However, learned facilitation was evident in both groups. MTL activity, indexed by blood flow changes measured with positron emission tomography, was correlated with facilitation in both groups but in opposite directions (greater MTL activity was related to less facilitation in AWARE subjects but more facilitation in UNAWARE subjects). Discrimination and reversal in AWARE subjects involved anterior medial, inferior prefrontal, and lateral occipital cortices. Furthermore, unique regional patterns of MTL functional connectivity were observed: AWARE subjects engaged dorsolateral prefrontal and lateral occipital cortices, whereas UNAWARE subjects showed a more spatially restricted network involving contralateral MTL regions and the thalamus. In the AWARE group, the MTL functional connectivity pattern overlapped with regions associated with facilitation and discrimination, but in UNAWARE subjects, the MTL pattern was related only to facilitation. These results suggest that the MTL and functional connected regions, including dorsolateral and medial prefrontal cortex, acted to link facilitation and discrimination patterns in AWARE subjects. Thus, the contribution of the MTL to learning and awareness is shaped by the pattern of interregional interactions, the neural context.

Key words: awareness; associative learning; prefrontal cortex; human; functional connectivity; PET; covariance

\section{Introduction}

Many contemporary theories in neurobiology emphasize the interactions among distributed brain regions as the key mechanism by which overt behavior and cognitive functions are produced (Mesulam, 1990; Bressler, 1995; McIntosh, 1999; Friston and Price, 2001). Recent neuroimaging evidence has demonstrated that various aspects of behavior are accompanied by changes in interregional interactions (McIntosh et al., 1996b; Buchel and Friston, 1997). Buchel et al. (1999a) observed that the relationship between dorsal and ventral cortical areas changed as participants learned a relationship between visual stimuli and their location. Importantly, this latter study demonstrated that individual differences in the changes of neural interactions were directly related to the subjects' rate of learning.

The basis for the present study comes from a differential sensory learning paradigm in which one tone predicted a visual event, and the other did not (McIntosh et al., 1999a). Half of the subjects in that study learned the stimulus relationships and half did not, and those that learned were the only subjects to profess awareness of the stimulus relationships. Activity changes in the

Received March 12, 2003; revised May 15, 2003; accepted May 16, 2003.

This study was supported by the Canadian Institutes of Health Research and the Natural Sciences and Engineering Research Council of Canada (A.R.M.).

Correspondence should be addressed to A. R. McIntosh, Rotman Research Institute of Baycrest Centre, 3560 Bathurst Street, Toronto, Ontario M6A 2E1, Canada. E-mail: mcintosh@psych.utoronto.ca.

Copyright $\odot 2003$ Society for Neuroscience $\quad$ 0270-6474/03/236520-09\$15.00/0 left prefrontal cortex (PFC) were observed only in AWARE subjects and depended on whether or not the tone predicted the visual stimulus (V). Prefrontal cortex activity was related to activity in auditory, visual, contralateral prefrontal cortices and basal ganglia only in the AWARE group. This finding, combined with strong relationships between activity in these regions and performance, provided evidence that an interacting system supported learning in the AWARE subjects.

In the present study, a similar learning protocol was used, except that the predictive value of the two tones was reversed midway through the experiment. As in the previous study, half of the participants were aware of the associations and learned the initial discrimination and reversal. The other participants were not aware of any relationship between the tones and visual stimuli. However, in this case, UNAWARE subjects did learn a simple tone-visual stimulus association. To further test the hypothesis that interactions among distributed brain regions support learning and awareness, we focused on large-scale patterns of functional connectivity (Friston, 1994) in the two groups. The behavioral responses provided a robust, objective measure of the group differences; thus, the relationship between brain activity and behavior was used as the principle means to identify functional connectivity patterns. Three aspects of these patterns are emphasized. First, activity in the medial temporal lobe (MTL) was differentially related to learning a general tone-visual association in both groups. This finding is surprising, given recent suggestions 
that the MTL is critical for learning with awareness (Clark and Squire, 1998; Manns et al., 2000). Second, the MTL was functionally connected with different brain regions in the two groups. Third, in the AWARE subjects, the pattern of MTL functional connectivity also included regions related to the associative, or learned, discrimination, such as dorsolateral and middle prefrontal cortex. These three outcomes suggest that, although certain regions may be critical for the expression of a particular behavior, their contribution can only be realized within the context of distributed interacting neural systems (McIntosh, 2001). We present the evidence for this claim below.

\section{Materials and Methods}

\section{Subjects}

Fourteen healthy right-handed subjects (seven males) between the ages of 19 and 35 (mean age, 23.3) participated in this study. All subjects were screened for any history of major medical, neurological, and psychiatric disorders and were provided informed consent. The experiment was conducted with approval from the Ethics Review Board of Baycrest Geriatric Centre, University of Toronto.

\section{Behavioral methods}

Two auditory stimuli (tones) and a visual stimulus were presented to subjects who were instructed to press a button on a computer mouse as quickly as possible when they saw the visual stimulus. They were also instructed to attend to the tones, and that either tone could precede the visual stimulus (Kimble and Perlmutter, 1970). The experiment was divided into two phases. In the first phase, one tone (T1; $1200 \mathrm{~Hz}$ pure tone) perfectly predicted $\mathrm{V}$; the second tone (T2; $600 \mathrm{~Hz}$ pure tone) was followed by V only $33 \%$ of the time. For the present purposes, we shall refer to the $100 \%$ predictive tone as " + " (S+) and the $33 \%$ predictive tone as "-" (S-; i.e., T1 + and T2 - for phase one). In the second phase, the predictability of the tones with respect to $\mathrm{V}$ reversed (i.e., $\mathrm{T} 1-, \mathrm{T} 2+$ ). All stimuli were presented for $500 \mathrm{msec}$. On paired trials, in which a tone preceded $\mathrm{V}$, there was a $300 \mathrm{msec}$ delay between the termination of the tone and the onset of $\mathrm{V}$. When a tone was presented by itself, or unpaired, there was an average of $8 \mathrm{sec}$ (range, $4-12 \mathrm{sec}$ ) before the next stimulus presentation.

Each phase of the experiment was divided into four 8-min blocks. In the middle of each block, a 1-min positron emission tomographic (PET) scan was obtained from each subject. Scans were designated S+ and Sand alternated across the experiment (phase 1: $\mathrm{T} 1+, \mathrm{T} 2-, \mathrm{T} 1+\mathrm{T} 2-$; phase $2: \mathrm{T} 2+, \mathrm{T} 1-, \mathrm{T} 2+, \mathrm{T} 1-$ ). During $\mathrm{S}+$ scans, five paired trials were presented, whereas $\mathrm{S}-$ scans consisted of five unpaired trials and five presentations of $\mathrm{V}$. The remaining trials within each block consisted of pseudo-random presentations: $6 \mathrm{~V}$ alone, 18 paired $\mathrm{S}+, 18$ unpaired $\mathrm{S}-$, and 6 paired $S-$ trials. Subjects were given a 4 -min break between each block. The entire procedure took $\sim 2 \mathrm{hr}$.

The visual stimulus and a fixation cross were presented in the center of a color video monitor that was positioned $\sim 60 \mathrm{~cm}$ from subject. The visual stimulus was a pattern of white concentric circles presented on a $50 \%$ gray background and subtended $12.5^{\circ}$ of visual angle. A white fixation cross was presented between trials. The tones were presented binaurally through earphones. Starting from $65 \mathrm{~dB}$, the amplitudes of the two tones were adjusted so that they were perceived to be equally loud by the experimenters. Stimulus presentation and collection of behavioral data were accomplished using Superlab for Windows (The Experimental Laboratory Software, version 1.03; Cedrus Corporation, San Pedro, CA).

Mean reaction times (RTs) were calculated over the interval before and during each scan for $\mathrm{V}$ when presented alone and when preceded by either tone and were used as an index of learning. The RT has been demonstrated to be a sensitive measure of conditioning in other associative learning paradigms (Critchley et al., 2002; Gottfried et al., 2002). Two aspects of RT changes were anticipated. First, it is well documented that subjects will respond more quickly to a visual stimulus when preceded by a tone, and that this facilitation grows as subjects learn (Hershenson, 1962; Schmidt et al., 1984; Perruchet, 1985; McIntosh et al., 1998). This learned facilitation is observed regardless of the predictive value of the tone (McIntosh et al., 1999a). Second, as we demonstrated previously (McIntosh et al., 1999a), subjects will sometimes respond more quickly when a tone is an S+ stimulus than when it is an S- stimulus, providing a behavioral indication of learned discrimination.

Subjects were given an open-ended hierarchical debriefing questionnaire at the end of the study. The purpose of the questionnaire was to determine whether a subject knew the relationship between the tones and visual stimuli. The questions started with general statements ("What did you think the experiment was about?") and then more specific questions about the tones were asked ("Did you use any strategies to help you respond to the visual stimulus? If so, what were they?" "Did you notice a relationship between the tones and visual stimulus? If so, what were they?"). Once the questionnaire was completed, subjects were debriefed further and paid for participating.

\section{PET scan protocol and image processing}

The details of our PET protocol and image processing have been described previously in full (McIntosh et al., 1998; Grady et al., 2001). Briefly, regional cerebral blood flow (rCBF) was measured during a 60sec scan after a bolus injection of $\left[\mathrm{O}^{15}\right]$ water via a catheter implanted in the left forearm. The PET images were corrected for head motion using AIR2.0 (Woods et al., 1993), spatially registered to an rCBF template that conformed to Talairach and Tournoux (1988) stereotaxic space, and smoothed with a $10 \mathrm{~mm}$ isotropic Gaussian filter using SPM99 (Statistical Parametric Mapping; Wellcome Department of Cognitive Neurology, London, UK) (Friston et al., 1995).

\section{Data analysis}

Behavior. Subjects were designated as either AWARE or UNAWARE at the end of the experiment on the basis of their responses during the debriefing. AWARE subjects were those who correctly stated the tonevisual associations. Subjects were designated as UNAWARE if they did not state an overt knowledge of any tone-visual associations and did not use the tones to guide their responses. Mean RT for paired T1, paired T2, and $\mathrm{V}$-alone trials was calculated within each of the eight blocks. Between-group analysis of the behavioral data was conducted using a repeated measures ANOVA comparing mean RT on the three trial types. Post hoc Newman-Keuls analysis was used to identify within-block RT differences.

Images. Partial least squares (PLS) analysis was used to identify distributed brain activity patterns related to behavior and MTL activity (McIntosh et al., 1996a; Della-Maggiore et al., 2000). A behavioral PLS analysis was used to identify distributed patterns of brain activity related to RT. A seed PLS analysis was used to assess the distributed functional connectivity patterns of the MTL.

The behavioral PLS procedure identifies voxels contributing to systematic brain-behavior correlations. Correlations between RT averaged within a block, and rCBF values at each voxel are first computed across subjects and within the scan. This produces one correlation map per RT measure per scan for each group. The correlation maps are combined into a single matrix and analyzed with singular value decomposition (SVD). The SVD produces mutually orthogonal latent variables (LVs), each consisting of a singular value, singular image, and correlation profile. The singular value indicates the strength of the covariance between behavior and all brain voxels. The singular images indicate which voxels most strongly covary with behavior across scans. The numerical weights within the singular image are called saliences and can be positive or negative. The singular image is multiplied by the raw images (dotproduct), producing a brain score for all subjects in each condition. The correlation of behavior with brain scores across subjects within each scan generates a profile that aids in interpreting the LV. If the correlation profiles show similarities across scans, salient areas in the singular image will have a similar correlation with RT across scans. If correlation differs between scans, the singular image will reflect this difference in brainbehavior correlation. The behavior PLS was performed on RT for T1+ and $\mathrm{T} 2+$ only. A separate analysis that included V-alone RT did not change the results reported here. The overall significance of the LVs was assessed using permutation tests, and reliability of image saliences and correlation profiles were assessed by bootstrap estimation of confidence intervals (McIntosh and Gonzalez-Lima, 1998; McIntosh et al., 1999a). 
The seed PLS procedure identifies voxels contributing to systematic correlations of a target "seed voxel" with the remaining voxels. In PET images, single voxels can act as regions of interest because of their rather large spatial autocorrelation (McIntosh et al., 1996b). Activity at the left MTL (LMTL) seed voxel was extracted for each subject in each group (Table 1). The procedure for seed PLS was identical to the behavior PLS, except one covariance image was created for each scan. This image contained the within-group covariation of LMTL rCBF with rCBF across the remainder of the brain and across subjects.

\section{Results}

\section{Behavioral analysis}

During the hierarchical debriefing, all

AWARE subjects stated that they noted a relationship between the two tones and target V. UNAWARE subjects noted no explicit relationships among the stimuli, nor used the tones to guide their responses. There were no demographic differences between groups.

Figure 1 plots the behavioral data for the two groups. For AWARE subjects, two effects were obvious in the data (Fig. 1, left). First, RT to V was faster on paired trials, in which a tone preceded V, relative to V-alone trials. Second, the magnitude of this RT facilitation depended on whether the tone was $\mathrm{S}+$ or $\mathrm{S}-$. The $S+/ S-$ differentiation was strong in both phases of the study, indicating AWARE subjects acquired the differential association and learned the reversal of contingencies. For UNAWARE subjects (Fig. 1, right), RT was also faster on paired trials compared with $\mathrm{V}$-alone trials, but there was no differentiation between the two tones. This facilitation became larger across blocks, indicating it was a learned (conditioned) facilitation. An unconditioned facilitation would not be expected to change across blocks (Gielen et al., 1983).

The impressions gained from Figure 1 were corroborated by ANOVA, with a significant group $\times$ trial type (T1, T2, V-alone $) \times$ block interaction $\left(F_{(14,168)}=2.74 ; p<0.001\right)$. There were no overall group differences in RT, and there were no differences in $\mathrm{V}$-alone RT between groups across the experiment. Post hoc analysis of the within-block RTs for the AWARE group indicated that V-alone RT was longer than T1 RT in each block, longer than T2 RT starting in block 3, and that the RT to an S+ tone was faster to an $S-$ tone in all blocks of training $(p<0.05$ or $p<0.01)$. In the UNAWARE group, the only effect was that $\mathrm{V}$-alone RT was longer than both T1 RT and T2 RT starting in block 3, with no differences between T1 RT and T2 RT. Thus, facilitation to both tones became stable in block 3 for both AWARE and UNAWARE subjects. Additionally, for both groups, an equivalent increase in $\mathrm{V}$-alone $\mathrm{RT}$ was seen with training, a feature commonly observed in conditioning paradigms using RT (Perruchet, 1985; McIntosh et al., 1998, 1999a). Across blocks, significant differences from initial V-alone RTs were seen only at the end of training, after the facilitation to both tones was established (AWARE: blocks 5, 7; UNAWARE: blocks 6, 8). Thus, this change likely reflects the implicit use of the tones to prepare for a response.

\section{Image analysis: behavior PLS}

Two strong brain-behavior patterns related to the general learned facilitation effect were identified. Behavior latent variable 1 (results not shown) identified a set of regions commonly related

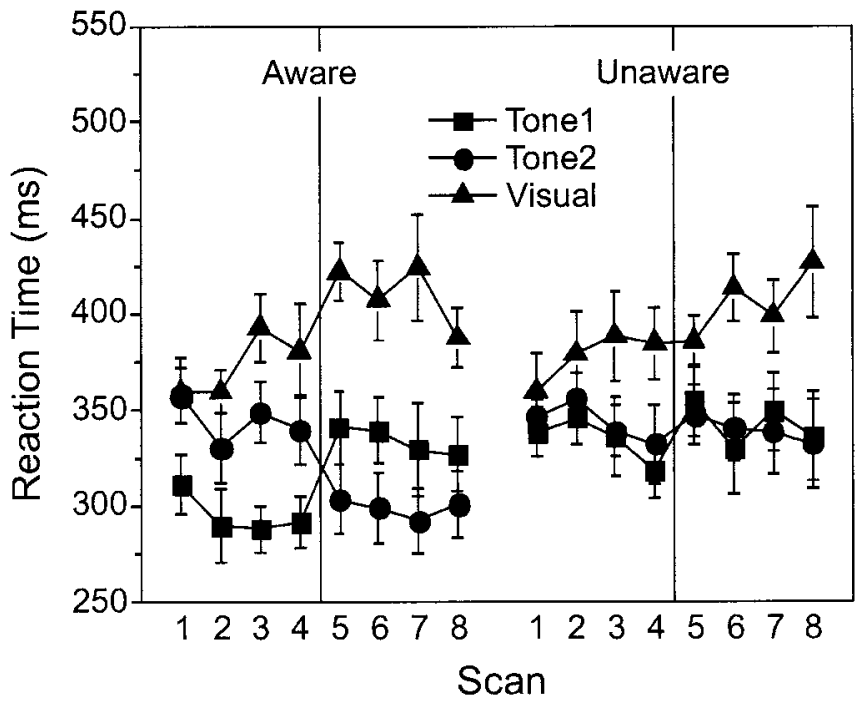

Figure 1. Mean RT ( \pm SEM) for AWARE (left) and UNAWARE (right) subjects. Both groups showed faster RTs on tone-visual trials (facilitation). AWARE subjects showed additional facilitation to the $S+$ in both phases, indicative of discrimination learning.

to learned facilitation in both groups. Across all scans, higher rCBF in bilateral thalamus, occipital, and middle temporal cortices was related to weaker facilitation (slower RTs), whereas greater activity in inferior temporal, anterior right prefrontal, and right inferior parietal cortices was related to stronger facilitation (faster RTs).

The second pattern behavior latent variable 2 (BehavLV2) identified regions in which the activity was differentially related to learned facilitation in the two groups. This is indicated by the opposing directions of the correlation in the profiles between groups (Fig. 2A,B; Table 1). Higher activity in the right parietal cortex, bilateral inferior prefrontal cortex, and right middle temporal cortex was associated with stronger facilitation in AWARE subjects and weaker facilitation in UNAWARE subjects. The most dominant region in this pattern was bilateral MTL, with more extensive involvement of LMTL. Higher MTL activity was related to stronger facilitation in UNAWARE subjects, whereas lower MTL activity was related to stronger facilitation in AWARE subjects.

Additional patterns of neural activity were related to the discrimination between $\mathrm{S}+$ and $\mathrm{S}-$ stimuli in the AWARE group (BehavLV3, BehavLV4; data not shown). The confidence intervals for the correlation profiles suggested that the pattern was 

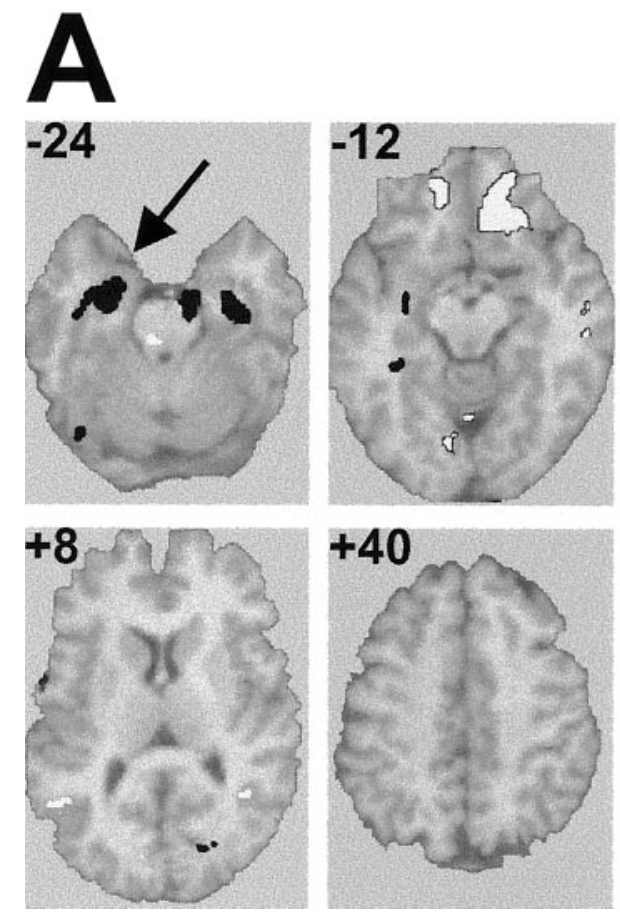

B

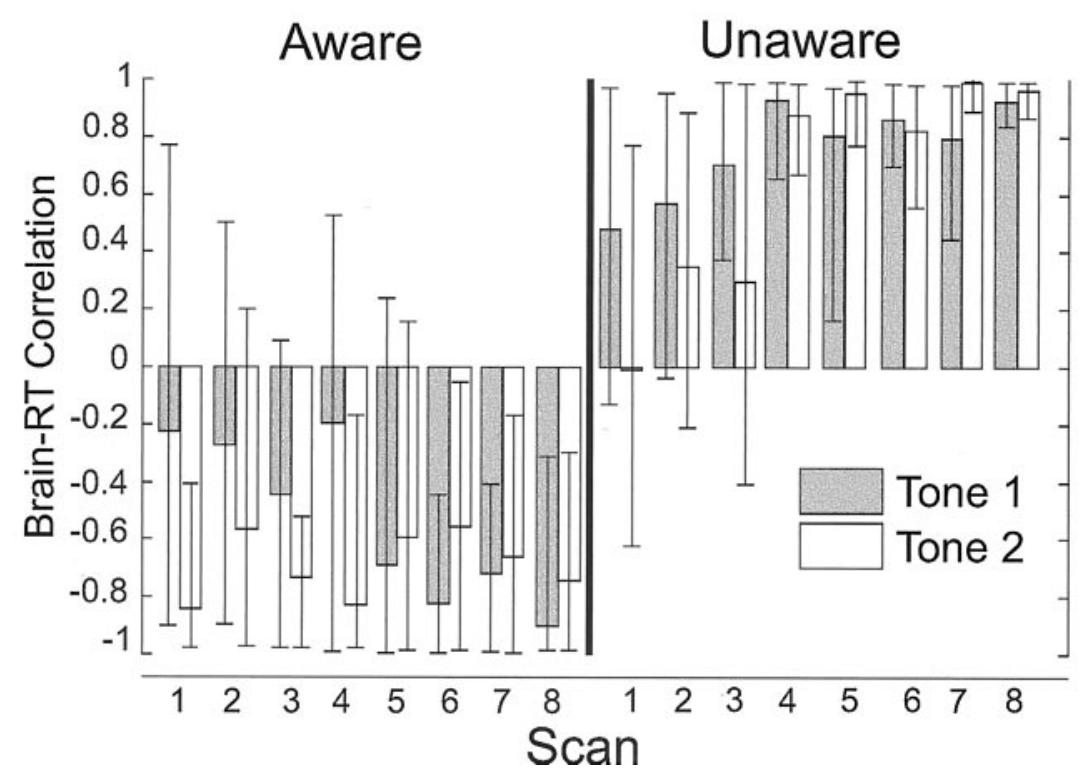

Figure 2. Second latent variable (BehavLV2) from the brain-behavior PLS analysis. A, Selected slices from the singular image overlaid on a structural magnetic resonance imaging (MRI), highlighting dominant regions that distinguished conditioned facilitation in AWARE and UNAWARE subjects. Slices are in the registration with the Talairach and Tournoux atlas (1988) and left is left in the slice. Activity in regions with positive brain saliences (white) was negatively correlated with RT in the AWARE group (stronger facilitation), positively correlated with RT on paired trials in the UNAWARE group (weaker facilitation), and vice versa for negative saliences (black). The arrow indicates the LMTL region used in the seed PLS analysis. B, Correlation profiles: correlations between $\mathrm{T} 1$ and T2 RTs and brain activity. Error bars indicate 95th percentile confidence interval around the correlation.

dominated by the response to the $\mathrm{S}+$ stimulus, which became more stable as AWARE subjects learned the significance of the predictor. The profiles for UNAWARE subjects showed reliable correlation for only the first three scans in the experiment and were equivalent for $\mathrm{T} 1$ and $\mathrm{T} 2$.

Separate behavior PLS analyses were conducted for AWARE and UNAWARE subjects to better appreciate their unique patterns of brain-behavior relationships. For each analysis, the first LV was highly significant and represented the regions involved in the general facilitation effects described above [AWARE_facilitation, $p \ll 0.001$, see Table S1 (available at www.jneurosci.org) for local maxima; UNAWARE_Facilitation1 (Fac1), $p \ll 0.001$, see Table S2 (available at www.jneurosci.org) for local maxima]. For the AWARE group only, the second LV was also significant $(p \ll 0.001)$ (Table S1). This pattern represented the additional facilitation seen on $S+$ trials in the learners, which became more statistically reliable with training (Table S1, AWARE_Discrimination; Fig. 3). The second pattern in the UNAWARE group was not significant by conventional thresholds $(p=0.08)$. It reflected stable, equivalent facilitation to $S+$ and $S-$ only in the first three scans (UNAWARE_Facilitation2; image not shown; Table S2). These secondary brain-behavior patterns will be discussed in more detail below.

\section{Image analysis: functional connectivity of left medial temporal lobe}

Both groups showed learned facilitation of RT; the LMTL was among a collection of regions that strongly differentiated facilitation in the AWARE and UNAWARE groups. Because of the speculation that the MTL is vital for learning with awareness, we focused in more detail on the LMTL region to explore its patterns of functional connectivity using seed PLS. LMTL activity measures were taken from the LMTL local maxima identified in the behavior PLS (Table 1). Unique patterns of LMTL functional connections were seen in the AWARE (SeedLV1: AWARE_LMTL, $p \ll 0.001$ ) and UNAWARE subjects (SeedLV2: UNAWARE_LMTL, $p \ll$ 0.001).

For the AWARE_LMTL pattern, correlation profiles were strong and stable across scans only in the AWARE group (Fig. 4) [see Table S3 (available at www.jneurosci.org) for local maxima]. Regions positively correlated with LMTL in the AWARE group included bilateral secondary visual cortices and bilateral auditory association cortices. Regions negatively correlated included the right middle temporal gyrus, inferior parietal lobe, and a large extent of bilateral dorsolateral prefrontal cortices. In contrast, the UNAWARE_LMTL pattern identified a LMTL network engaged only in the UNAWARE subjects (Fig. 5; Table S3). This network showed positive correlations between LMTL and the bilateral hippocampal gyrus, bilateral temporal cortex, and left secondary visual cortex, and showed inverse relationships between LMTL and the right inferior prefrontal cortex, left auditory association cortex, and bilateral thalamus.

\section{Image analysis: LMTL functional connections interact with behavioral networks}

In both the behavior and seed PLS, common areas were identified. What is noteworthy is how this overlap manifested for the two groups. In the AWARE group, regions from both the AWARE_facilitation pattern and the AWARE_Discrimination pattern were identified in the AWARE_LMTL pattern (Figs. 2-4, slices $+8,+40)$. In the UNAWARE group, strong regional overlap was noted only between the UNAWARE_Facilitation 1 pattern and the UNAWARE_LMTL pattern (Figs. 2, 5, slice -24). Thus, the LMTL was functionally connected with regions in- 

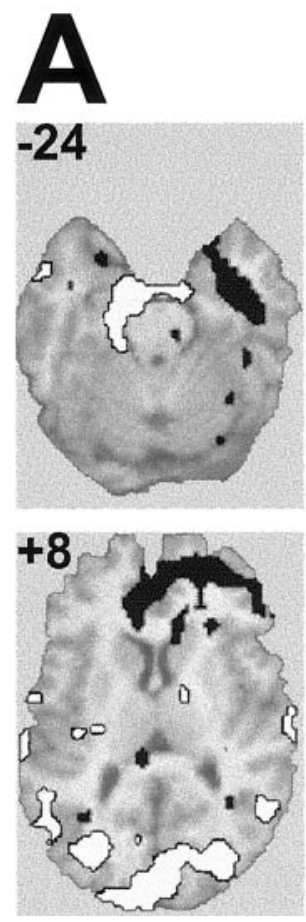
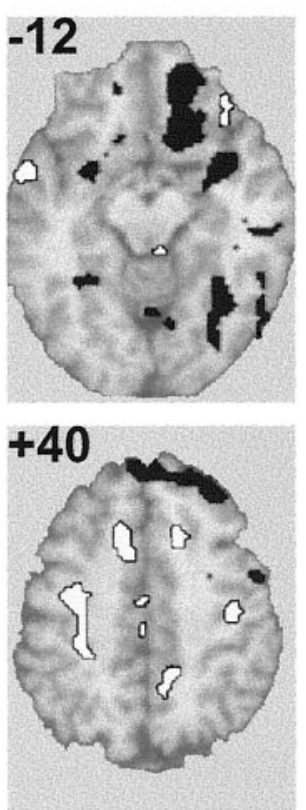

$B$

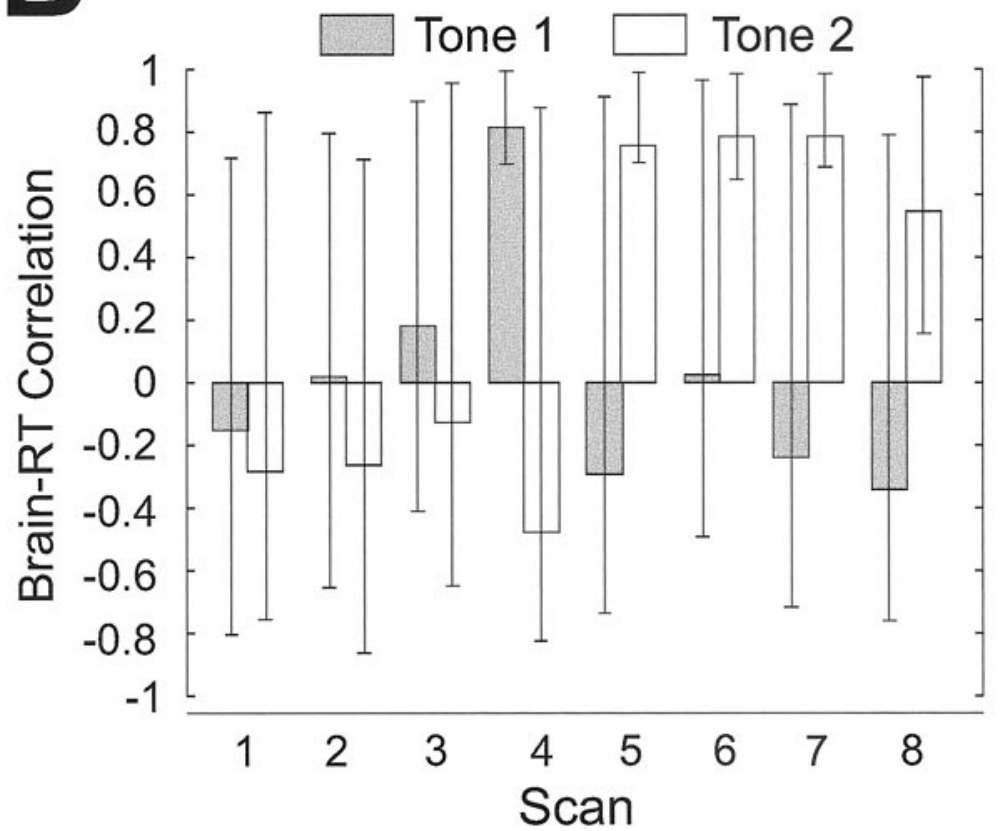

Figure 3. Second latent variable from the behavior PLS analysis in the AWARE group (AWARE_Discrimination). A, Selected slices from the singular image overlaid on a structural MRI, highlighting dominant regions. Increased activity in regions with negative saliences (black) and decreased activity in regions with positive saliences (white) were related to faster RT on $S+$ trials. B, Correlation profiles: correlation between $S+R T$ and brain activity became stable just before the reversal and remained strong across the remaining blocks. Error bars indicate 95 th percentile confidence interval around the correlation.
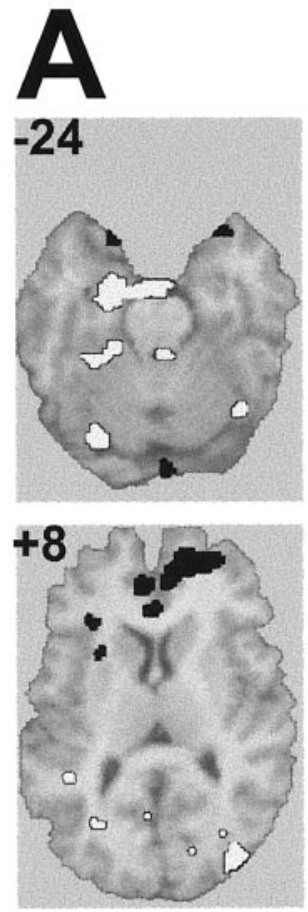
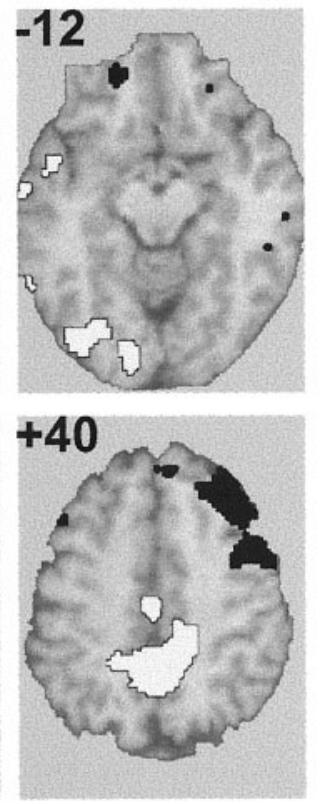

Aware

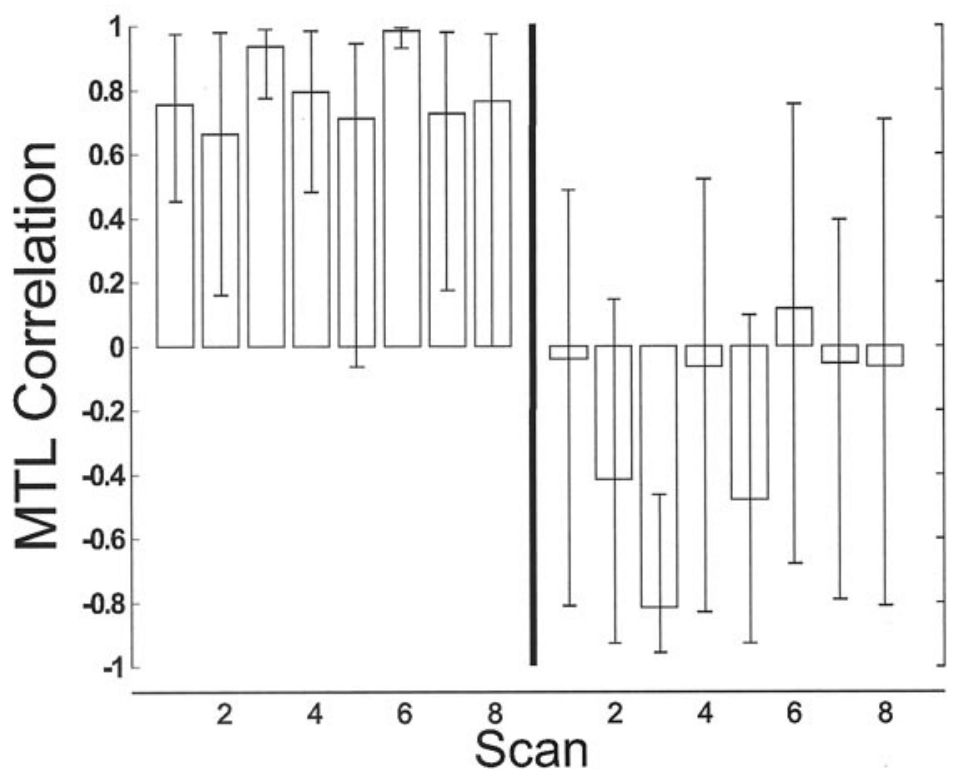

Figure 4. First latent variable from the between-group LMTL seed PLS analysis (SeedLV1). A, Selected slices from the singular image overlaid on a structural MRI, highlighting regions related to LMTL activity in AWARE subjects. Regions with positive brain saliences (white) were positively correlated with LMTL activity, and regions with negative brain saliences (black) were negatively correlated with LMTL activity. B, Correlation profiles: LMTL- brain activity correlations were reliable for AWARE subjects only as indicated by the error bars on the correlation profiles.

volved in facilitation and discrimination in the AWARE subjects but only with a single set of regions involved in facilitation in the UNAWARE subjects. This finding raises the possibility that the LMTL in AWARE subjects may have been acting as a link be- tween the brain regions associated with facilitation and discrimination.

To assess whether the voxels in the LMTL connectivity pattern were also functionally connected to voxels in the behavior net- 
A
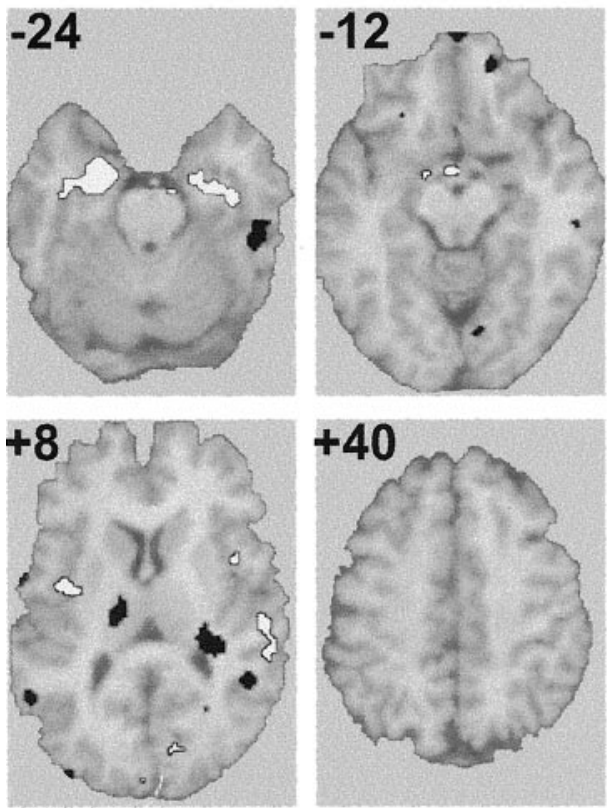

B

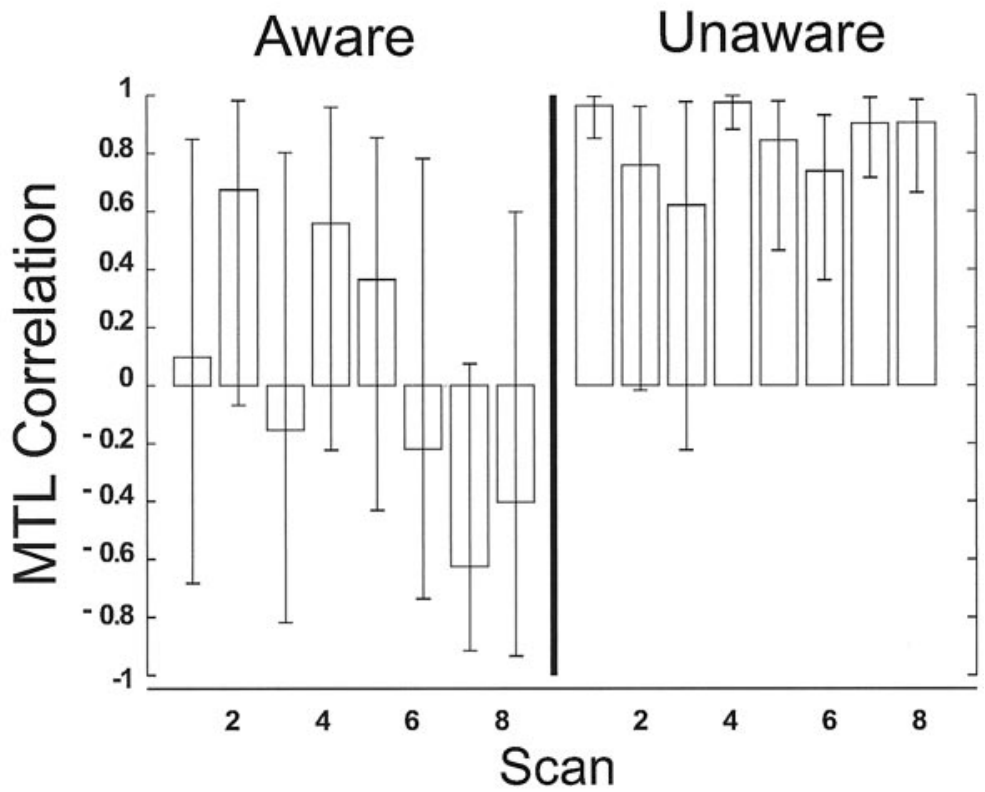

Figure 5. Second latent variable from the between-group LMTL seed PLS analysis (SeedLV2). A, Selected slices from the singular image overlaid on a structural MRI, highlighting regions related to LMTL activity in UNAWARE subjects. Regions with positive brain saliences (white) were positively correlated with LMTL activity, and regions with negative brain saliences (black) were negatively correlated with LMTL activity. B, Correlation profiles: LMTL-brain activity correlations were reliable for UNAWARE subjects only.
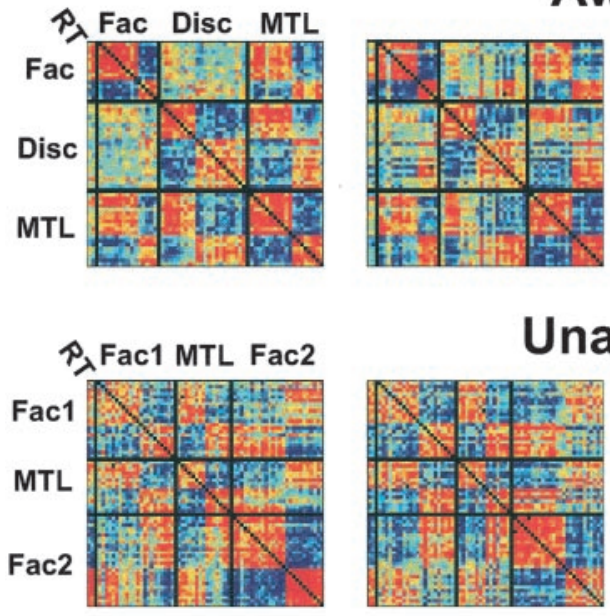

\section{Aware}
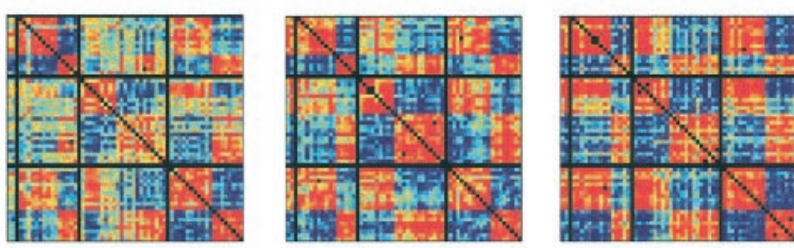

Unaware

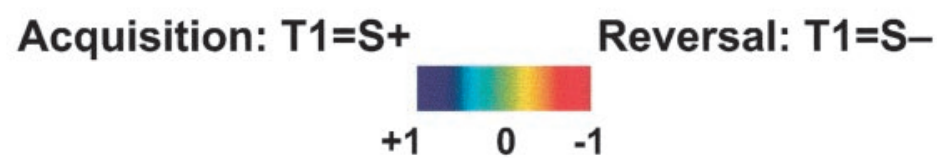

Figure 6. Pseudo-colored scan-specific correlation matrices for the first two scans in acquisition and the first two scans after reversal. Top plots, AWARE; bottom plots, UNAWARE. For AWARE subjects, correlations among voxels from the AWARE_Facilitation (Fac) (Table S1), AWARE_Discrimination (Disc) (Table S1), and AWARE_LMTL seed PLS (MTL) (Table S3, SeedLV1) patterns are shown. For UNAWARE subjects, correlations among voxels from the UNAWARE_Facilitation1 (Fac1) (Table S2), UNAWARE_LMTL seed PLS (MTL) (Table 4, SeedLV2), and UNAWARE_Facilitation2 (Fac2) (Table S2) patterns are shown. For both groups, the first two columns (RT) indicate the correlation of all voxels with $\mathrm{T} 1$ and $\mathrm{T} 2 \mathrm{RT}$. Black lines divide each matrix into sectors emphasizing the different correlation patterns (e.g., the correlation between Fac and Disc patterns is at the intersection of the Fac and Disc columns). Red, Positive correlation; blue, negative correlation. works, $\mathrm{rCBF}$ correlations were produced using the dominant voxels observed in the within-group behavior PLS (Tables S1, S2) and the voxels identified in the LTML seed PLS (Table S3). For the AWARE group, correlations among the voxels from AWARE_facilitation, AWARE_Discrimination, and AWARE_LMTL were generated (Fig. 6, top). For the UNAWARE group, correlations from UNAWARE_Facilitation1 and UNAWARE LMTL were obtained (Fig. 6, bottom). Correlations with the voxels from the secondary facilitation pattern in the UNAWARE group (Table S2, UNAWARE_Facilitation2) are also included for completeness. Matrices along the diagonal represent the correlations among voxels within each of the PLS patterns, whereas the matrices on the offdiagonal are the correlations between the patterns (the matrices are symmetric). The first two columns of each matrix contain the correlation of each voxel with RT to each tone. Black lines in the larger matrix divide the plots into sectors highlighting the correlations within and between patterns. In the interest of brevity, only the correlations for the first two scans in acquisition and the first two scans after reversal (scans 5 and 6) are shown.

The figure shows strong correlations among voxels identified for the facilitation 
patterns in both groups (top left sector of the matrices), which is consistent with the observation that both groups showed strong learned facilitation across the entire study. For the AWARE group, the voxels from the AWARE_facilitation and AWARE_Discrimination patterns were strongly correlated with AWARE_LMTL voxels across the experiment (facilitation vs MTL, discrimination vs MTL sectors, respectively), whereas correlations between AWARE_Discrimination and AWARE_facilitation (discrimination vs facilitation sector) showed a linear increase with training $[p<0.008$; significance of the changes in the correlation matrices across scans were assessed using a permutation test of a linear regression on the matrix norms (largest eigenvalue) (McIntosh et al., 1999b)]. This pattern suggests that the coherence among all three patterns increased with learning. In the UNAWARE group, regions from UNAWARE_LMTL became more strongly correlated with regions from UNAWARE_Facilitation1 (Facl vs MTL sector; $p<0.028$ ). Correlations among UNAWARE_Fac2 voxels with the UNAWARE_LMTL and UNAWARE_Facilitation1 voxels were strong early in the experiment but diminished as the experiment progressed ( $p<0.038, p<$ 0.056 , respectively). To summarize, the correlations among voxels from behavior PLS and the LMTL seed PLS were on the whole strong and tended to increase with learning in the AWARE group, whereas in the UNAWARE group, only the correlations between the regions related to learned facilitation (faciliation1) maintained a strong correlation with regions functionally connected to the LMTL.

\section{Discussion}

We demonstrated that under identical training conditions, only subjects that were aware of the stimulus contingencies acquired differential associations, but all subjects showed conditioned facilitation. This offered an opportunity to explore whether differences in the neural substrates for behavior might explain differences in awareness in the two groups. Three aspects of the current results are important. First, both AWARE and UNAWARE subjects learned something about the stimulus relationships. UNAWARE subjects expressed this as a general facilitation of RT when a tone preceded the visual target. AWARE subjects also showed learned facilitation but showed additional facilitation when tones predicted the visual stimulus $(\mathrm{S}+)$. This additional facilitation to $\mathrm{S}+$ occurred both before and after the reversal of stimulus contingencies, indicative of associative discrimination. Second, the brain-behavior analysis suggested that both groups engaged the MTL, including the hippocampal formation. Although opposite relationships of MTL activity with learning were found in the two groups, the strength of the relationship indicates a role for the MTL in learning without awareness. Third, largescale functional connectivity of the LMTL with the rest of the brain was strikingly different in the two groups. In AWARE subjects, the regions functionally connected with LMTL were themselves correlated with regions that related to learned facilitation and discrimination, suggesting a potential link between the two learned behaviors. In UNAWARE subjects, the LMTL functional connections were limited to regions involved in learned facilitation. This leads to the possibility that the nature of MTL interactions with other brain regions may determine how learning proceeds and whether it is accompanied by awareness.

\section{Large-scale functional connectivity}

The finding that the correlations among distributed patterns (between latent variables) changed depending on awareness and what was learned (Fig. 6) deserves additional discussion. The statistical analysis used to extract the patterns of brain-behavior relationships does so by identifying mathematically orthogonal latent variables. For the present discussion, these orthogonal patterns were treated as representing separate neural systems with different effects on behavior. It is equally plausible that a combination of regions across these orthogonal patterns may represent a single system having multiple effects on behavior. This is consistent with the results from the AWARE group analyses. The behavioral results in the AWARE subjects included two effects: conditioned facilitation and conditioned discrimination. The behavior PLS showed that these two behavioral effects were supported by spatially distinct patterns of brain activity. However, the interactions between these two patterns increased with learning, as evidenced by the increase in the correlations among the sets of regions (Fig. 6, left). Thus, the present results demonstrate that the neural systems that are statistically independent may be strongly interacting. In the case of the UNAWARE group, there appeared to be only one reliable behavioral effect, and thus a single discrete pattern of brain activity. Interactions with other systems that were temporarily recruited became weak or effectively zero (e.g., UNAWARE_Facilitation2) (Fig. 6, bottom).

\section{Medial temporal lobe functional connectivity}

Evidence from both lesion and electrophysiological studies shows that the hippocampus and related MTL structures are critical for trace conditioning (which is similar to our present sensory learning paradigm) and other forms of conditioning that depend on awareness (Solomon et al., 1986; James et al., 1987; Buchel et al., $1999 b)$. In humans, MTL involvement in awareness has been documented in both lesion and neuroimaging studies (Hamann and Squire, 1997). However, hippocampal cell activity has been consistently related to acquisition of conditioned responses in delay conditioning (Laroche et al., 1987; Miller and Steinmetz, 1997), which need not be accompanied by awareness. This leads to the possibility that the nature of hippocampal interactions with other brain regions may govern what is learned and whether learning is accompanied by awareness (Manns et al., 2000). Our data suggest that distinct patterns of hippocampal interactions underlie some of the differences in what was learned between AWARE and UNAWARE subjects.

The patterns of functional connectivity identified for the AWARE group included a large region of dorsolateral prefrontal cortex (Fig. 3). The extensive involvement of the PFC is intriguing, because it has been associated with awareness in previous studies (Lumer and Rees, 1999; McIntosh et al., 1999a). The correlation between the PFC and LMTL was negative, a pattern we observed previously in perceptual (Della-Maggiore et al., 2000) and episodic (McIntosh et al., 1997) memory tasks. Similar inverse relationships between the PFC and MTL have been reported in rodent electrophysiological studies of spatial working memory (Laroche et al., 2000). The inverse relationships of the LMTL and $\mathrm{PFC}$ in the present study provide a possible substrate for learning in the AWARE subjects and may partly account for the differences in awareness and learning between groups. For the UNAWARE group, the strongest functional connections remained within the MTL, extending bilaterally. These strong positive interactions within temporal cortices, in the absence of frontal involvement, resemble findings from animal studies, in which strong entorhinal-hippocampal interactions are seen early in learning, followed by hippocampal-prefrontal interactions (Laroche et al., 2000). The spatial extent of the MTL involvement in the UNAWARE group suggests that strong entorhinal-hippocampal interactions did not extend to the prefrontal cortex. 
On the basis of present results and evidence from other work discussed here, we suggest that the vital neural substrate for learning with awareness lies in the pattern of interactions between the MTL and prefrontal cortex.

The relationship of LMTL activity to learned facilitation was opposite in the AWARE and UNAWARE subjects, with stronger facilitation in the AWARE subjects supported by decreased LMTL activity. Diminution of MTL involvement with learning has been seen for the orienting response that accompanies the presentation of the conditional stimulus (CS) (Deadwyler et al., 1981), in category learning in which the hippocampal response to learned relevant stimuli was smaller than for unlearned or irrelevant stimuli (Aizenstein et al., 2000), and in classification learning in which MTL activity was initially high and then decreased as learning proceeded (Poldrack et al., 2001). In this latter study, learning-related changes in MTL functional connectivity were also noted.

\section{Functional connectivity and neural context}

A key feature distinguishing the involvement of the MTL in the two groups was the neural context in which the medial temporal lobe participated (McIntosh, 1999). Neural context refers to the notion that the functional role of a region depends on the other regions to which it is related. Electrophysiological data indicate that contextual influences occur for individual neurons and cell populations (Kozlov and Shabaev, 2000; Worgotter and Eysel, 2000). At the systems level, a region may show similar activity patterns across tasks, yet be part of different networks, producing different behavioral outcomes (Bressler and Kelso, 2001). The present results indicate differences in large-scale interactivity involving the MTL, which contributed to learning in the presence and absence of awareness. Complementary findings indicate that distinct MTL functional networks can also support similar behavioral outcomes (Della-Maggiore et al., 2000) and may account for episodic memory performance in patients with MTL damage (Maguire et al., 2000). In the present study, strong MTL interactivity was found in UNAWARE subjects showing a simple form of learning (Fig. 6), despite the fact that learning without awareness does not appear to rely on MTL integrity.

When considered from the perspective of neural context, it may be that richly interconnected regions like the MTL enable transitions between behavioral states by interacting first with one set of regions and then another. In a sense, the MTL may act to catalyze or facilitate the transition from implicit to explicit knowledge (Moscovitch, 1995) or from a state of "unconditional stimulus expectancy" to "CS expectancy" (Lovibond and Shanks, 2002; Shanks and Lovibond, 2002). The MTL would be engaged regardless of the nature of the behavioral state, but a move to an explicit state would not occur without a particular pattern of functional connectivity. Some evidence of this was seen in the present study, because the interactions became stronger with training among the AWARE_Facilitation and AWARE_Discrimination networks, both of which were strongly related to the LMTL functional connections. Thus, the group differences in LMTL interactions and behavior offer preliminary evidence that the MTL acts as a behavioral catalyst. MTL functional connections in the UNAWARE group did not engage more spatially distant regions, such as the PFC and sensory cortices, which may be a necessary condition for learning with awareness. In more extreme instances of MTL damage, certain behavioral transitions would obviously not occur. By acting as a catalyst, the MTL could participate in several different behavioral functions, such as perception and simple conditioning, but would be critical in those instances when it enables the transition from one pattern of functional connections and behavioral state to another. This proposal expands the role of the MTL and emphasizes that, although a region may be critical for the expression of a given behavior, the exact expression of behavior comes from unique combinations of neural processes encompassing several brain regions. In other words, the specific pattern of neural interactions shapes the exact nature of the behavior expressed.

\section{References}

Aizenstein HJ, MacDonald AW, Stenger VA, Nebes RD, Larson JK, Ursu S, Carter CS (2000) Complementary category learning systems identified using event-related functional MRI. J Cognit Neurosci 12:977-987.

Bressler SL (1995) Large-scale cortical networks and cognition. Brain Res Rev 20:288-904.

Bressler SL, Kelso JAS (2001) Cortical coordination dynamics and cognition. Trends Cognit Sci 5:26-36.

Buchel C, Friston K (1997) Modulation of connectivity in visual pathways by attention: cortical interactions evaluated with structural equation modeling and fMRI. Cereb Cortex 7:768-778

Buchel C, Coull JT, Friston KJ (1999a) The predictive value of changes in effective connectivity for human learning. Science 283:1538-1541.

Buchel C, Dolan RJ, Armony JL, Friston KJ (1999b) Amygdala-hippocampal involvement in human aversive trace conditioning revealed through eventrelated functional magnetic resonance imaging. J Neurosci 19:10869-10876.

Clark RE, Squire LR (1998) Classical conditioning and brain systems: the role of awareness. Science 280:77-81.

Critchley HD, Mathias CJ, Dolan RJ (2002) Fear conditioning in humans: the influence of awareness and autonomic arousal on functional neuroanatomy. Neuron 33:653-663.

Deadwyler SA, West MO, Robinson JH (1981) Entorhinal and septal inputs differentially control sensory-evoked responses in the rat dentate gyrus. Science 211:1181-1183.

Della-Maggiore V, Sekuler AB, Grady CL, Bennett PJ, Sekuler R, McIntosh AR (2000) Corticolimbic interactions associated with performance on a short-term memory task are modified by age. J Neurosci 20:8410-8416.

Friston K (1994) Functional and effective connectivity: a synthesis. Hum Brain Mapp 2:56-78.

Friston KJ, Price CJ (2001) Dynamic representations and generative models of brain function. Brain Res Bull 54:275-285.

Friston KJ, Ashburner J, Frith CD, Pline J-B, Heather JD, Frackowiak RSJ (1995) Spatial registration and normalization of images. Hum Brain Mapp 2:165-189.

Gielen SC, Schmidt RA, Van den Heuvel PJ (1983) On the nature of intersensory facilitation of reaction time. Percep Psychophys 34:161-168.

Gottfried JA, O'Doherty J, Dolan RJ (2002) Appetitive and aversive olfactory learning in humans studied using event-related functional magnetic resonance imaging. J Neurosci 22:10829-10837.

Grady CL, McIntosh AR, Beig S, Craik FI (2001) An examination of the effects of stimulus type, encoding task, and functional connectivity on the role of right prefrontal cortex in recognition memory. NeuroImage 14:556-571.

Hamann SB, Squire LR (1997) Intact perceptual memory in the absence of conscious memory. Behav Neurosci 111:850-854.

Hershenson M (1962) Reaction time as a measure of intersensory facilitation. J Exp Psychol 63:289-293.

James GO, Hardiman MJ, Yeo CH (1987) Hippocampal lesions and trace conditioning in the rabbit. Behav Brain Res 23:109-116.

Kimble GA, Perlmutter LC (1970) The problem of volition. Psychol Rev 77:361-384.

Kozlov AP, Shabaev VV (2000) Analysis of the dynamics of interneuronal functional connections during conditioned reflex activity. Neurosci Behav Physiol 30:625-634.

Laroche S, Neuenschwander-el Massioui N, Edeline JM, Dutrieux G (1987) Hippocampal associative cellular responses: dissociation with behavioral responses revealed by a transfer-of-control technique. Behav Neural Biol 47:356-368.

Laroche S, Davis S, Jay TM (2000) Plasticity at hippocampal to prefrontal cortex synapses: dual roles in working memory and consolidation. Hippocampus 10:438-446.

Lovibond PF, Shanks DR (2002) The role of awareness in Pavlovian condi- 
tioning: empirical evidence and theoretical implications. J Exp Psychol Anim Behav Process 28:3-26.

Lumer ED, Rees G (1999) Covariation of activity in visual and prefrontal cortex associated with subjective visual perception. Proc Natl Acad Sci USA 96:1669-1673.

Maguire EA, Mummery CJ, Buchel C (2000) Patterns of hippocampalcortical interaction dissociate temporal lobe memory subsystems. Hippocampus 10:475-482.

Manns JR, Clark RE, Squire LR (2000) Parallel acquisition of awareness and trace eyeblink classical conditioning. Learn Mem 7:267-272.

McIntosh AR (1999) Mapping cognition to the brain through neural interactions. Memory 7:523-548.

McIntosh AR (2001) Towards a network theory of cognition. Neural Network 13:861-876.

McIntosh AR, Gonzalez-Lima F (1998) Large-scale functional connectivity in associative learning: interrelations of the rat auditory, visual and limbic systems. J Neurophysiol 80:3148-3162.

McIntosh AR, Bookstein FL, Haxby JV, Grady CL (1996a) Spatial pattern analysis of functional brain images using partial least squares. NeuroImage 3:143-157.

McIntosh AR, Grady CL, Haxby JV, Ungerleider LG, Horwitz B (1996b) Changes in limbic and prefrontal functional interactions in a working memory task for faces. Cereb Cortex 6:571-584.

McIntosh AR, Nyberg L, Bookstein FL, Tulving E (1997) Differential functional connectivity of prefrontal and medial temporal cortices during episodic memory retrieval. Hum Brain Mapp 5:323-327.

McIntosh AR, Lobaugh NJ, Cabeza R, Bookstein FL, Houle S (1998) Convergence of neural systems processing stimulus associations and coordinating motor responses. Cereb Cortex 8:648-659.

McIntosh AR, Rajah MN, Lobaugh NJ (1999a) Interactions of prefrontal cortex related to awareness in sensory learning. Science 284:1531-1533.
McIntosh AR, Sekuler AB, Penpeci C, Rajah MN, Grady CL, Sekuler R, Bennett PJ (1999b) Recruitment of unique neural systems to support visual memory in normal aging. Curr Biol 9:1275-1278.

Mesulam MM (1990) Large-scale neurocognitive networks and distributed processing for attention, language, and memory. Ann Neurol 28:597-613.

Miller DP, Steinmetz JE (1997) Hippocampal activity during classical discrimination-reversal eyeblink conditioning in rabbits. Behav Neurosci 111:70-79.

Moscovitch M (1995) Recovered consciousness: a hypothesis concerning modularity and episodic memory. J Clin Exp Neuropsychol 17:276-290.

Perruchet P (1985) Expectancy for airpuff and conditioned eyeblinks in humans. Acta Psychol 58:31-44.

Poldrack RA, Clark J, Pare-Blagoev EJ, Shohamy D, Creso Moyano J, Myers C, Gluck MA (2001) Interactive memory systems in the human brain. Nature 414:546-550.

Schmidt RA, Gielen SC, van den Heuvel PJ (1984) The locus of intersensory facilitation of reaction time. Acta Psychol 57:145-164.

Shanks DR, Lovibond PF (2002) Autonomic and eyeblink conditioning are closely related to contingency awareness: reply to Wiens and Ohman (2002) and Manns et al., (2002). J Exp Psychol Anim Behav Process 28:38-42.

Solomon PR, Vander Schaaf ER, Thompson RF, Weisz DJ (1986) Hippocampus and trace conditioning of the rabbit's classically conditioned nictitating membrane response. Behav Neurosci 100:729-744.

Talairach J, Tournoux P (1988) Co-planar stereotaxic atlas of the human brain. New York: Thieme Medical Publishers.

Woods RP, Mazziotta JC, Cherry SR (1993) MRI-PET registration with automated algorithm. J Comput Assist Tomogr 17:536-546.

Worgotter F, Eysel UT (2000) Context, state and the receptive fields of striatal cortex cells. Trends Neurosci 23:497-503. 